\title{
A Multi-Aperture Scintillation Sensor for Dome A, Antarctica
}

\author{
Hualin Chen ${ }^{1,2,3,4}$ Chong Pei $^{1,2,3}$ and Xiangyan Yuan ${ }^{1,2}$ \\ ${ }^{1}$ National Astronomical Observatories/Nanjing Institute of Astronomical Optics \& Technology, \\ Chinese Academy of Sciences, Nanjing 210042, China \\ ${ }^{2}$ Key Laboratory of Astronomical Optics \& Technology, Nanjing Institute of Astronomical \\ Optics \& Technology, Chinese Academy of Sciences, Nanjing 210042, China \\ ${ }^{3}$ Graduate University of Chinese Academy of Sciences, Beijing 100049, China \\ ${ }^{4}$ email: hualinchen@niaot.ac.cn
}

\begin{abstract}
Site-testing measurements by the Australian group has already shown that Dome $\mathrm{C}$ on the Antarctic plateau is one of the best ground-based astronomical sites. Furthermore, Dome A, the Antarctic Kunlun Station, as the highest point on Antarctic inland plateau, where a Chinese Antarctic scientific expedition team first reached in 2005, is widely predicted to be an even better astronomical site by the international astronomical community. Preliminary site-testing carried out by the Center for Antarctic Astronomy (CAS) also confirms Dome A as a potential astronomical site. Multi-aperture scintillation sensors (MASS) can measure the seeing and isoplantic angle, the turbulence profile, etc., which are very important site-testing parameters that we urgently need. The MASS site testing at Dome A is presented here, and includes the method of processing data and the hardware for the extreme conditions of Dome A, Antarctica.
\end{abstract}

Keywords. Antarctic Kunlun Station, Dome A, Scintillation, Seeing, Isoplanatic angle, Turbulence profile.

\section{Theory}

Theory of wave propagation shows: Scintillation $s^{2} \sim \int C_{n}^{2} \cdot Q(h) \cdot d h$, Seeing $r_{0} \sim$ $\left[\int C_{n}^{2} \cdot d h\right]$, Isoplanatic angle $\theta_{0} \sim\left[\int C_{n}^{2} \cdot h^{5 / 3} \cdot d h\right]^{3 / 5}$, where $Q(h)$ is weight function $(\mathrm{WF})$. It depends on the receiving aperture geometry, light spectrum and the assumed theory of turbulence.

\section{Data processing}

Scintillation indices calculation and their errors. A set of instantaneous scintillation indices (SI), including normal, differential and differential exposure scintillation indices, are calculated using raw photo counts from a given number of channels during the base time $1 \mathrm{~s}$. To obtain correct SIs from raw data, we must take into account non-linearity of photo counters, subtract the contribution from photo noise, and extrapolate the indices to zero-exposure time for removing the bias caused by finite exposure time, make correction for background include dark count of PMTs and light from the sky.

Weighting functions. The weighting function (WF) depends on spatial turbulence spectrum and on the shape and size of receiving aperture. In addition, some de-correlation of scintillation within the bandpass is also expected. So, for an altitude $h$, polychromatic weighting functions table for both normal and differential SIs are pre-computed.

Profile restoration. A low-resolution turbulence profile (TP) restoration method is adopted which is fitting the measured indices to a model with only a few layers, then 


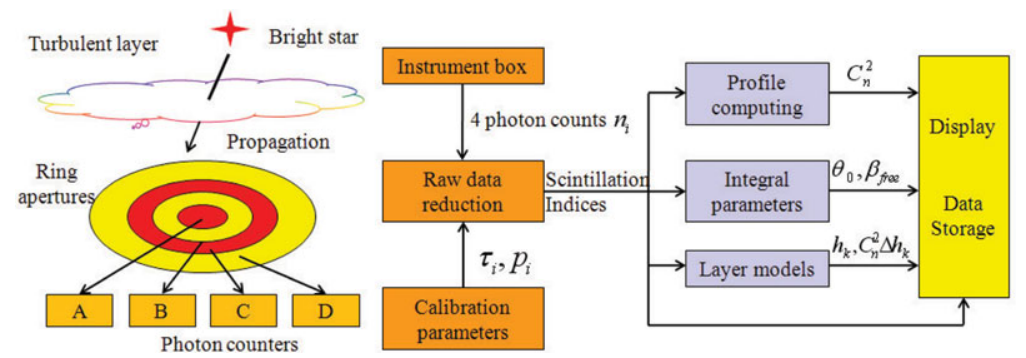

Figure 1. Left: principle of turbulence profile measurement with the MASS. Right: organization of the data processing in the MASS.

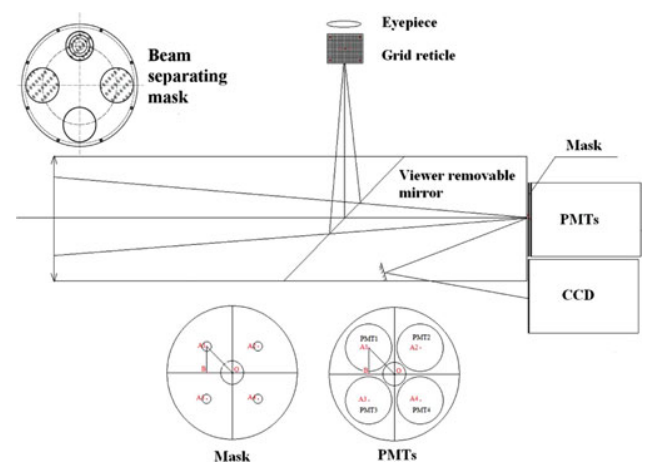

Figure 2. Simplified layout of the MASS instrument.

restoring these thick slabs of turbulence. Both fixed-layers with pre-defined altitudes and floating-layers with only three layers located at any altitudes restoration techniques are used. In fitting models to the data, we search for model parameters that minimize the merit function.

Seeing, isoplantic angle and atmospheric time constant. Because each SI is an integral over altitude of $C_{n}^{2}$ multiplied by some WF. Thus, any linear combination of SIs corresponds to the linear combinations of WFs. If the linear combinations of WFs approximates $h^{0}$ or $h^{3 / 5}$, then seeing or isoplanatic angle can be estimated from liner combinations of SIs directly, without profile restoration. The atmosphere time constant can be estimated from differential exposure scintillation index as: $\tau_{\mathrm{de}}=\mathrm{KT}\left[\sigma_{\mathrm{DE}}^{2}\right]^{3 / 5}$.

\section{Hardware}

A beam separating mask in the telescope entrance pupil separates the star light into six clusters. Two clusters image the star on the CCD, functioning as a differential image motion monitor (DIMM) measuring the whole atmosphere seeing. The other four clusters converge on PMTs through the telescope respectively. The PMTs output a pulse sequence corresponding to the photon sequence counting for the scintillation index, measuring the free atmosphere seeing, the isoplanatic angle and the turbulence profile. Low temperature protection is used for printed circuit boards, PMTs, CCDs and other important models. Thw data processing computer is in a nearby instrument house. The combination of DIMM and MASS is simple and robust, and should work at Dome A.

\section{References}

http://www.ctio.noao.edu/ atokovin/profiler/. 